\title{
Theoretical Molecular Docking Study of the Structural Disruption of the Viral 3CL-Protease of COVID19 Induced by Binding of Capsaicin, Piperine and Curcumin Part 1: A Comparative Study with Chloroquine and Hydrochloroquine Two Antimalaric Drugs
}

\author{
Lenin A. Gonzalez-Paz ( $\sim$ lgonzalezpaz@gmail.com) \\ Laboratorio de Genética y Biología Molecular (L.G.B.M). Departamento de Biología. Facultad Experimental de Ciencias (F.E.C). Universidad del Zulia (L.U.Z), \\ Venezuela.

\section{Carla A. Lossada} \\ Laboratorio de Caracterización Molecular y Biomolecular- Centro de Investigación y Tecnología de Materiales (CITeMA)- Instituto Venezolano de \\ Investigaciones Científicas (I.V.I.C), Venezuela. \\ Luis S. Moncayo \\ Universidad Católica de Cuenca, Unidad Académica de Salud y Bienestar. Farmacia, Laboratorio de Microbiología, Ecuador. \\ Freddy Romero \\ Center for Traslational Medicine. Thomas Jefferson University, USA. \\ J. L. Paz \\ Departamento de Física. Escuela Politécnica Nacional, Ladrón de Guevara, Ecuador. \\ Joan Vera-Villalobos \\ Escuela Superior Politécnica del Litoral, Facultad de Ciencias Naturales y Matemáticas. Departamento de Química y Ciencias Ambientales. Laboratorio de \\ Análisis Químico Instrumental (LAQUINS). \\ Aleivi E. Pérez \\ Laboratorio de Microbiología General (L.M.G). Departamento de Biología. Facultad Experimental de Ciencias (F.E.C). Universidad del Zulia (L.U.Z). Venezuela \\ Emesto San-Blas \\ Laboratorio de Protección Vegetal, Centro de Estudios Botánicos y Agroforestales, Instituto Venezolano de Investigaciones Científicas (I.V.I.C), Venezuela. \\ Ysaias J. Alvarado \\ Laboratorio de Caracterización Molecular y Biomolecular- Centro de Investigación y Tecnología de Materiales (CITeMA)- Instituto Venezolano de \\ Investigaciones Científicas (I.V.I.C), Venezuela.
}

\section{Research Article}

Keywords: COVID-19, natural products, molecular docking, molecular dynamics

Posted Date: April 6th, 2020

DOI: https://doi.org/10.21203/rs.3.rs-21206/v1

License: (9) (7) This work is licensed under a Creative Commons Attribution 4.0 International License. Read Full License 


\section{Abstract}

The global pandemic caused by infections of the new coronavirus (COVID-19) makes it necessary to find possible less toxic and easily accessible therapeutic agents. In this study, we used strategies docking and molecular dynamics to analyze phytochemical compounds against FDA-approved antimalarial drugs recommended for the treatment of COVID-19. The evaluation was performed with the docking scores MolDock Score and Rerank Score calculated by Molegro Molecular. The DockThor server was used to generate the complexes and myPresto for the dynamic studies. Preliminary results suggested that piperine, capsaicin, and curcumin have the best docking scores and that they are capable of promoting structural changes in the viral protease by inducing folding of the enzyme. Curcumin and capsaicin bring the enzyme to a more compact conformational state compared to the native state, compared to chloroquine. Even though, it is unknown if these induced changes in protease are related to any inhibitory effect observed both in vitro and in vivo for any of these compounds. Further studies on the mechanisms of action of these compounds of interest are required, as well as experimental demonstrations. However, these results are interesting because they can serve as a starting point for subsequent experimental or/and in silico studies based on chemical structure-activity relationships taking these small molecules and their possible derivatives.

\section{Introduction}

The first case of the new Coronavirus was reported December 30, 2019, in Wuhan city, Hubei province, P. R. China (Xu et al., 2020), and by the beginning of March the cases amounted to 113,702 confirmed and 4,012 deaths worldwide (Jiang et al., 2020) necessitating the rapid development and approval of a vaccine not yet available to date (Jiang et al., 2020). However, it has been suggested that the selection of FDA-approved drugs with potential antiviral activity against related viruses may yield promising results (Chang et al., 2020; Contini, 2020), reporting that older antimalarial drugs, such as chloroquine, have the ability to interact strongly in silico with viral 3CL-protease (Chang et al.,2020) recently crystallographed (Chang et al.,2020; Liu et al., 2020), and with an important inhibitory effect in vitro and in vivo (Gautret et al., 2020; Wang et al., 2020). Reason why its use has been recommended in China and USA for the treatment of COVID-19 (Devaux et al., 2020). This despite the fact that its safety has been questioned and severe adverse effects have been reported (Wang et al., 2020; Kaisari and Borruat, 2020). In this sense, various bioactive compounds of plant origin have also been in silico studied why could they be used as alternative medicines or to develop new drugs against COVID-19 with fewer adverse effects (Khaerunnisa et al., 2020; Qamar et al., 2020; Sharma and Kaur, 2020; Chandel et al.,, 2020; Adem et al.,, 2020; Gentile et al.,, 2020; Sun et al.,, 2020). But little has been reported on the use of the MolDock molecular docking algorithm for the 3CL-protease study (Adem et al.,, 2020), in which the docking score function is an extension of the linear part potential (PLP) that includes new hydrogen bonds and electrostatic terms to improve coupling precision (Thomsen and Christensen, 2006; Wang, 2020). This being the first study to report the structural disturbance that the natural compounds considered in this study can induce in the viral protease of COVID-19. An approach that has only been applied to antiviral drugs (Alamri et al.., 2020). Therefore, in the present study was performed a theoretical study with MolDock molecular docking algorithm to obtain information on the interaction of various phytochemical compounds described with potential antiviral activity directed at the COVID-19 protease.

\section{Methods}

Docking Screening: In this study, the 3CL-protease structure (PDB ID: 6LU7) was obtained from the RCSB Protein Data Bank, which was recently released on February 5th, 2020 (Chang et al., 2020). Based on a literature survey, we tested eighteen phytochemical compounds with reported antiviral properties including, Allicin (Xie et al.,2020), Bornyl acetate (Sun et al., 2020), Capsaicin (Tang et al., 2020), Carvacrol (Bansal et al., 2020), Catechol (Mishra et al., 2020), Cinnamic acid (Silva et al., 2020), Curcumin (Bonfim et al., 2020), Eucalyptol (Patra et al., 2020), Gallic acid (Arsianti et al., 2020), Geraniol (Wei et al., 2020), Linalool (Del Bosco et al., 2020), Nicotinic acid (Corona et al., 2020), Phytic acid (Narayanaswamy and Esa 2018), Piperine (Chandani et al., 2019), Quercetin (Nile et al., 2020; Lopes et al., 2020; Aggarwa et al., 2020), Resorcinol (Lee et al., 2019), Terpinen-4-ol (Patra et al., 2020) and Viridiflorene (Shayeganmehr et al., 2018). Additionally, we have used as molecular docking controls, two FDA-approved old antimalarial drug, Chloroquine and Hydroxychloroquine (an analogue of chloroquine) recommended for treating COVID-19 patients (Gautret et al., 2020). Molecular structures of all compounds can be seen in the table S1 of information supplementary.

We downloaded the 3D structure of each drug from the PubChem database in Structure-data file (SDF) format (Gautret et al., 2020) and Online SMILES Translator (https://cactus.nci.nih.gov/translate/) to convert SDF to PDB format. To simulate binding affinity between protein and ligands, the complexes were built in Dockthor (https://dockthor.Incc.br/v2/) using the flexibility algorithm and the search parameters blind docking by programming 25 runs and $10^{6}$ evaluations for each ligand (Dos Santos et al., 2020). Taken the most favored position to be analyzed with Molegro Molecular Viewer 2019_7.0.0 software, MolDock Score and Rerank Score functions were calculated (Thomsen and Christensen, 2006). We also used myPresto ligand docking utilities to dock the selected drugs to the protease under default settings. myPresto is tailored for a single process execution with a single GPU (Graphics Processing Unit), in order to optimize the enhanced conformational sampling methods (Kasahara et al., 2016).

Molecular dynamics (MD) simulation: MD simulations were first performed for a docking hit for two purposes: (1) studying the relative stability of the ligand residing in the binding pocket; (2) sampling a set of conformations for MM (molecular mechanics). A MD system consisted of one copy of COVID-19 protease, one copy of docked ligand and about $50 \mathrm{Na}^{+}$and $\mathrm{Cl}^{-}$ions. The whole system was neutralized. For a protein-ligand complex, the $\mathrm{MD}$ system was first relaxed through a series of minimization procedures. There were three phases for a MD simulation: the relaxation phase, the equilibrium phase, and the sampling phase, as recommended (Wang, 2020). In the relaxation phase, the simulation system was heated up progressively to $250 \mathrm{~K}$. A 2-picosecond MD simulation was performed without any restraints or constraints. In the next equilibrium phase, the system was equilibrated at $298 \mathrm{~K}, 1$ bar for 2 ps. Finally, a 2 ps MD simulation was performed at $298 \mathrm{~K}, 1$ bar to produce NTP (constant temperature and pressure) ensembles. Simulations were also made at 4 ps. All MD simulations and the additional settings were performed using myPresto program. 
Complementary Analysis: The Molinspiration server was used to calculate the partition coefficient (milogP) and calculation of bioactivity scores (Cheminformatics, M. 2020; Reena Roy et al., 2020). Analysis of variance of a factor (ANOVA) was used as a statistical model to establish differences between the comparative parameters analyzed, including the structural changes generated by the thermodynamically most favored ligands. Tukey was applied as a means separation test and hypothesis test. All calculations were carry out using the IBM SPSS-23 program.

\section{Results And Discussion}

In Table 1, we list the results of the coupling of 20 ligands with the $3 \mathrm{CL}$ protease. These scores, which are the original raw results of the coupling tools, represent the relative binding affinity obtained with MolDock, and differ from those reported using Autodock 4.2, with the Lamarckian Genetic Algorithm for curcumin, allicin, and quercetin, in terms of their positions against inhibitors like lopinavir (Khaerunnisa et al., 2020). But they correspond to those reported by another author for curcumin and piperine versus chloroquine using Autodock 4.2.6 (Mohammad et al., 2020). 17\% of the phytochemical compounds presented more favorable docking energies than hydroxychloroquine and chloroquine for both scoring functions considered. For scoring functions MolDock Score y Rerank Score, piperine (-62.467 and $-54.751 \mathrm{kcal} / \mathrm{mol})$, capsaicin $(-55.224$ and $-52.694 \mathrm{kcal} / \mathrm{mol})$ and curcumin $(-54.727 \mathrm{and}-46.143 \mathrm{kcal} / \mathrm{mol})$ have the lowest docking score and thermodynamically more favorable, respectively; even outperforming the results of hydroxychloroquine ( -48.737 and $39.491 \mathrm{kcal} / \mathrm{mol}$ ) and chloroquine $(-46.582$ and $-37.804 \mathrm{kcal} / \mathrm{mol})$, respectively (see Table 1 and Figure 1$)$, the two antimalarial drugs currently in use, and outperforming the results getting with Lopinavir ( -31.959 and $-30.65 \mathrm{kcal} / \mathrm{mol}$, respectively) a potent HIV-1 protease inhibitor not considered in this study (Capparelli et al., 2005), 44\% of the phytochemical presented more favorable docking energies than Lopinavir according to the MolDock Score, and $28 \%$ by Rerank Score, every found effective in treating COVID-19 patients. These results seen in Table.1 are promising if we consider that the MolDock molecular docking algorithm, based on a heuristic search that combines differential evolution with a cavity prediction algorithm, in which the MolDock docking score function is an extension of the PLP that includes new hydrogen bonds and electrostatic terms improves docking precision, by introducing a re-classification scoring function, which identifies the most promising docking solution of the solutions obtained by the docking algorithm, a strategy that has allowed to increase the docking precision surpassing other algorithms used in studies related to the protease associated with COVID-19 (Thomsen and Christensen, 2006; Wang, 2020). We therefore choose piperine, capsaicin and curcumin for further investigation with the filter MM. Doing a comparative analysis with hydroxychloroquine and chloroquine (see Table 1).

Table 1. Results of the virtual screening was done on the crystal structure of COVID 19 main 3CL-protease (PDB code 6LU7). Showing 18 phytochemical compounds with reported antiviral properties, and additionally as molecular docking controls, two FDA-approved old antimalarial drug for potential treatment, Chloroquine and Hydroxychloroquine (an analogue of chloroquine) recommended in treating COVID-19 patients. Phytochemicals with better scores than controls are highlighted in bold.

\begin{tabular}{|c|c|c|c|c|}
\hline Compound Name & $C I D$ & $\begin{array}{c}\text { MolDock Score } \\
(\mathrm{kcal} / \mathrm{mol})\end{array}$ & $\begin{array}{c}\text { Rerank Score } \\
(\mathrm{kcal} / \mathrm{mol})\end{array}$ & Interactions \\
\hline Piperine & 638024 & -62.467 & -54.751 & Arg4 (HB, SI) \\
\hline Curcumin & 969516 & -55.224 & -52.694 & Lys5 (SI) - Val125 (HB) - Asp289 (HB) - Lys137 (HB) \\
\hline Capsaicin & 1548943 & -54.727 & -46.143 & Asp $153(\mathrm{HB})-$ Lys102 (SI) \\
\hline Hydroxychloroquine* & 3652 & $-48.7 \overline{3} 7$ & -39.491 & Lys5 (HB, SI) - Glu288 (HB, SI) \\
\hline Chloroquine* & 2719 & -46.582 & -37.804 & Lys5 (HB, SI) \\
\hline Allicin & 65036 & -36.104 & -31.062 & Lys137 (HB, SI) \\
\hline Viridiflorene & 10910653 & -33.190 & -28.051 & ND \\
\hline Geraniol & 637566 & -31.520 & -25.941 & Lys102 (HB, SI) - Asp153 (HB, SI) \\
\hline Linalool & 6549 & -31.451 & -29.211 & Phe3 (HB) - Arg4 (SI) \\
\hline Gallic acid & 370 & -30.290 & -30.044 & Asp155 (HB) - Asp153 (HB) - Tyr154 (HB, SI) \\
\hline Carvacrol & 10364 & -28 & -27.170 & Glu240 (HB) - Ile200 (SI) \\
\hline Quercetin & 5280343 & -25 & -29.412 & Glu240 (HB) - Asp245 (HB) - Gln107 (SI) - Ile200 (SI) \\
\hline Phytic acid & 890 & -24 & 742 & Lys5 (HB) - Glu288 (HB) - Gly138 (HB) - Lys137 (HB, \\
\hline Resorcinol & 5054 & -21.341 & -19.120 & Asp289 (HB) - Glu290 (HB) - Lys137 (SI) \\
\hline Cinnamic acid & 444539 & -20.229 & -19.724 & Asp153 (HB) - Ser158 (HB) - Lys102 (SI) \\
\hline Bornyl acetate & 6448 & -19.237 & -22.198 & Arg298 (HB, SI) \\
\hline Eucalyptol & 2758 & -18.800 & -20.821 & ND \\
\hline Catechol & 2 & & -18 & Ser10 (HB) - Glu14 (HB) - Gly11 (SI) \\
\hline Nicotinic acid & 938 & -15 & 967 & Arg153 (HB) - Ser158 (HB) \\
\hline Terpinen-4-ol & 11230 & & -16.437 & Lys102 (HB) - Asp153 (HB) \\
\hline
\end{tabular}

*, FDA-approved old antimalarial drug recommended for treating COVID-19 patients; HB, hydrogen bonds; SI, Steric Interactions; ND; not determined.

On the other hand, for the promising coupling results, we performed molecular dynamics (MD) simulations using the myPresto software package and the results obtained are shown in Table.2.

Table 2. MD simulations using the myPresto software package and calculation of the partition coefficient and bioactivity scores with the Molinspiration server. After calculating the trajectories of the different positions of the atoms of the ligand-protein complexes as a function of time, the microscopic behavior of the protein in the presence of each ligand was simulated, as well as the bioaccumulation and bioactivity of the ligands.

\begin{tabular}{|c|c|c|c|c|c|c|c|c|}
\hline Compound Name & $\begin{array}{c}M W \\
(g / \mathrm{mol})\end{array}$ & $\begin{array}{c}E \text {-potential } \\
\text { (kcal/mol) }\end{array}$ & $\begin{array}{c}\text { Time }(p s e c) / E- \\
\text { potential }\end{array}$ & $\begin{array}{c}E \text {-Total } \\
(\mathrm{kcal} / \mathrm{mol})\end{array}$ & $\begin{array}{c}\text { Time (psec)/ E- } \\
\text { Total }\end{array}$ & $\begin{array}{l}\log \\
K_{\text {ow }}\end{array}$ & $\begin{array}{c}P \\
\text { Score }\end{array}$ & $\begin{array}{c}E \\
\text { Score }\end{array}$ \\
\hline Piperine & 285.34 & -8960 & 1.1 & -2800 & 2.8 & 3.33 & -0.10 & 0.04 \\
\hline Curcumin & 368.40 & -8930 & 1.1 & -2800 & 2.0 & $3.29^{\mathrm{a}}$ & -0.14 & 0.08 \\
\hline Capsaicin & 305.40 & -9000 & 1.3 & -2800 & 1.2 & $3.04^{\mathrm{b}}$ & -0.02 & 0.07 \\
\hline Hydroxychloroquine & 335.90 & -8900 & 1.3 & -2800 & 3.6 & 4.00 & 0.12 & 0.15 \\
\hline Chloroquine & 319.90 & -8930 & 1.1 & -2800 & 1.6 & $4.63^{\mathrm{c}}$ & 0.05 & 0.11 \\
\hline
\end{tabular}


P Score, protease inhibitor score (-0.50 - 1.50) Cheminformatics, M. 2020; E Score, enzyme inhibitor score (-0.50 - 1.50$)$ Cheminformatics, M. 2020; $\log K_{\mathrm{ow}}, 2$ - 5 (Bhal, 2007); ${ }^{\text {, }}$ EPA, U. (2010). Estimation Program Interface (EPI) Suite; b, LaHann, T. R., DeKrey, L. J., and Tarr, B. D. (1989). Capsaicin analgesia: predictions based on physico-chemical properties. In Proceedings of the Western Pharmacology Society (Vol. 32, pp. 201-204); ', Leo, A., and Hoekman, D. H. (1995). Exploring QSAR. American Chemical Society.

After calculating the trajectories of the different positions of the atoms of the ligand-protein complexes as a function of time, to simulate the microscopic behavior of the protein in the absence and presence of each ligand, we found that all the 6 main compounds generate interaction systems thermodynamically stable and similar to each other in terms of total energy $(\Delta \mathrm{G} \leq-2800 \mathrm{kcal} / \mathrm{mol})$. However, it is important to note that while the protease in the native state reaches its least-energy structure at 1.6 ps (see Table 2 and Figure 3), the three candidate phytochemical compounds may induce structural disturbances that affect the thermodynamic stability of the enzyme around of $1.1-1.3$ ps of the simulation, by like antimalarial drugs and commercial viral protease inhibitor. Specifically, capsaicin was the compound with which the most thermodynamically stable conformation was obtained at 1.3 ps, and piperine and curcumin, generated less stable structures than the native one in much less time (1.1 ps). These results show that the studied phytochemical compounds could be able to induce large structural fluctuations with respect to the native structure, generating changes in the thermodynamic stability of the enzyme in very early stages of the docking in terms of potential energy, similar to the tested drugs (see Table 2 and Figure 3 ).

In relation to the molecular properties and structural characteristics studied in these compounds, and which are of interest for the search for possible pharmacological objectives, it was determined that all the phytochemical ligands have a good partition coefficient (log $\left.K_{\text {ow }}: 3.04-3.29\right)$, and although they are below what was observed with antimalarials, these results suggest that the natural compounds studied are capable of bioaccumulating with a high coefficient (Bhal, 2007). Piperine and curcumin being the phytochemicals with the highest bioaccumulation capacities (Table 2). Additionally, the potential bioactivity of these compounds was studied, by means of the scoring obtained from the comparison of the phytochemicals within a calculated distribution of activity scores for protease inhibitor ligands and other enzymatic targets compared to scores for approximately 100,000 average molecules similar to drugs. The score allows the efficient separation of active and inactive molecules, finding that like antimalarials, who obtained the highest scores, all phytochemicals can be potential protease inhibitors, with capsaicin showing the best inhibition score for proteases among phytochemicals, while for curcumin the best prediction was obtained for enzyme inhibition in general (see Table 2).

Additionally, we were able to determine that all the ligands considered for the MD analyzes are capable of inducing structural disturbances in the viral protease $(3 \mathrm{CL})$ with a statistically significant difference in its folding or unfolding effects of the enzyme $(p<0.001)$ using as reference the distances between the residues of Arg4 and Gly138 (see Figure 2), arbitrarily chosen because they are approximately $16 \AA$ apart from each other and at a distance of approximately 2 $\AA$ from almost all the ligands (except for capsaicin, which presents a thermodynamically more favorable docking in a cavity that bets on predicted for the rest of the ligands).

These results are promising because they show the existence of known bioactive compounds of plant origin that could be used as model drugs in more exhaustive theoretical or/and experimental studies based in structure-activity relationship to develop new drugs against COVID-19. Especially, since it has been shown that compounds such as piperine can serve for the transport of bioactives of plant origin (Izgelov et al., 2020). A very important aspect because it has been reported that the active sites of SARS coronavirus proteins showing important differences in both shape and size and therefore are not compatible with the COVID-19 docking site, which may affect the efficacy of reused drugs proposed for treatment (Chang et al., 2020; Bzowka et al., 2020). Therefore, we are conducting a second phase of this study aimed at comparative analysis of thermodynamic, structural and conformational disturbances that are capable of inducing the compounds of interest considered in this study, including the contrast with protease inhibitor drugs.

\section{Conclusion}

Based in bioinformatics tools in this study was obtained that curcumin, capsaicin and piperine strongly binding to 3CL-protease of COVID-19 in comparison to the two antimalaric drugs and promote important structural changes in this viral protease, inducing folding of the enzyme. We propose that these three compounds could be candidates as model drugs for experimental and theoretical studies to evaluate their possible biological activity. In this sense, we recommend more studies on the mechanisms of action of these compounds of interest, as well as experimental demonstrations of the possible antagonistic effect in vitro on COVID-19.

\section{References}

Adem, S.; Eyupoglu, V.; Sarfraz, l.; Rasul, A.; Ali, M. 2020. Identification of Potent COVID-19 Main Protease (Mpro) Inhibitors from Natural Polyphenols: An in Silico Strategy Unveils a Hope against CORONA. Preprints, 2020030333.

Aggarwal, M., Leser, G and Lamb, R. 2020. Repurposing papaverine as an antiviral agent against influenza viruses and paramyxoviruses. Journal of Virology. 94(6), e01888-19.

Alamri, M. A.; Tahir ul Qamar, M.; Alqahtani, S. M. 2020. Pharmacoinformatics and Molecular Dynamic Simulation Studies Reveal Potential Inhibitors of SARSCoV-2 Main Protease 3CLpro. Preprints, 2020020308.

Arsianti, A., Bahtiar, A., Fadilah, F., Wangsaputra, V., Paramita, R., Azizah, N and Kakiuchi, K. 2020. Synthesis, haracterization, and cytotoxicity evaluation of gallic acid nanoparticles towards breast T47D cancer cells. Pharmacognosy Journal, 12(2).

Bzowka, M., Mitusinska, K., Raczynska, A., Samol, A., Tuszynski, J. A., and Gora, A. 2020. Molecular Dynamics Simulations Indicate the COVID-19 Mpro Is Not a Viable Target for Small-Molecule Inhibitors Design. bioRxiv. 
Bansal, A., Jan, I and Sharma, N. 2020. Anti-phytoviral activity of carvacrol vis-a-vis cauliflower mosaic virus (CaMV). Proceedings of the national academy of sciences, India section B: Biological Sciences, 1-8.

Capparelli, E., Holland, D., Okamoto, C., Gragg, B., Durelle, J., Marquie-Beck, J and HNRC Group. 2005. Lopinavir concentrations in cerebrospinal fluid exceed the $50 \%$ inhibitory concentration for HIV. Aids, 19(9), 949-952.

Chandel, V.; Raj, S.; Rathi, B.; Kumar, D. 2020. In Silico Identification of Potent COVID-19 Main Protease Inhibitors from FDA Approved Antiviral Compounds and Active Phytochemicals through Molecular Docking: A Drug Repurposing Approach. Preprints, 2020030349.

Bhal, S. 2007. LogP-Making sense of the value. Advanced chemistry development, Toronto, ON, Canada, 1-4.

Bonfim, C., Monteleoni, L., Calmon, M., Cândido, N., Provazzi, P., Lino, V and Melli, P. 2020. Antiviral activity of curcumin-nanoemulsion associated with photodynamic therapy in vulvar cell lines transducing different variants of HPV-16. Artificial Cells, Nanomedicine, and Biotechnology, 48(1), 515-524.

Chandani, S., Thorat, P., Nanda, R and Chitlange, S. 2019. Docking of phytoconstituents of Cynodon dactylon on NS2B NS3 protease domain of dengue virus. Research Journal of Pharmacy and Technology, 12(12), 5865-5870.

Chang, Y., Tung, Y., Lee, K., Chen, T., Hsiao, Y., Chang, H and Shih, S. 2020. Potential therapeutic agents for COVID-19 based on the analysis of protease and RNA polymerase docking. preprints.org.

Cheminformatics, M. 2020. Molinspiration. Web-enabled software for large-scale calculation of molecular properties and database searches, Free online molecular descriptor calculations.

Contini, A. 2020. Virtual screening of an FDA approved drugs database on two COVID-19 coronavirus proteins. chemrxiv.org.

Corona, A., Onnis, V., Del Vecchio, C., Esposito, F., Cheng, Y and Tramontano, E. 2020. 2-(Arylamino)-6-(trifluoromethyl) nicotinic acid derivatives: New HIV-1 RT dual inhibitors active on viral replication. Molecules, 25(6), 1338.

Del Bosco, S., Abbate, L., Mercati, F., Napoli, E and Ruberto, G. 2020. Essential oils in citrus. In the Citrus Genome (pp. 211-223). Springer, Cham.

Devaux, C., Rolain, J., Colson, P and Raoult, D. 2020. New insights on the antiviral effects of chloroquine against coronavirus: what to expect for COVID-19?. International Journal of Antimicrobial Agents, 105938.

Dos Santos, K., Guedes, I., Karl, A and Dardenne, L. 2020. Highly flexible ligand docking: Benchmarking of the DockThor program on the LEADS-PEP proteinpeptide dataset. Journal of Chemical Information and Modeling. 60(2), 667-683

Gautret, P., Lagier, J., Parola, P., Meddeb, L., Mailhe, M., Doudier, B and Honoré, S. 2020. Hydroxychloroquine and azithromycin as a treatment of CoVID-19: results of an open-label non-randomized clinical trial. International Journal of Antimicrobial Agents, 105949.

Gentile, D.; Patamia, V.; Scala, A.; Sciortino, M. T.; Piperno, A.; Rescifina, A. 2020. Inhibitors of SARS-CoV-2 Main Protease from a Library of Marine Natural Products: A Virtual Screening and Molecular Modeling Study. Preprints, 2020030372.

Gomez, D., Huber, K and Klumpp, S. 2019. On protein folding in crowded conditions. The Journal of Physical Chemistry Letters, 10(24), 7650-7656.

Izgelov, D., Domb, A. J., and Hoffman, A. 2020. The Effect of Piperine on Oral Absorption of Cannabidiol following Acute vs. Chronic Administration. European Journal of Pharmaceutical Sciences, 105313.

Jiang, F., Deng, L., Zhang, L., Cai, Y., Cheung, C and Xia, Z. 2020. Review of the clinical characteristics of coronavirus disease 2019 (COVID-19). Journal of General Internal Medicine, 1-5.

Khaerunnisa, S.; Kurniawan, H.; Awaluddin, R.; Suhartati, S.; Soetjipto, S. 2020. Potential Inhibitor of COVID-19 Main Protease (Mpro) From Several Medicinal Plant Compounds by Molecular Docking Study. Preprints, 2020030226.

Kaisari, E and Borruat, F. 2020. Keeping an Eye on Hydroxychloroquine Retinopathy. Klinische Monatsblätter für Augenheilkunde. Efirst.

Kasahara, K., Ma, B., Goto, K., Dasgupta, B., Higo, J., Fukuda, I and Nakamura, H. 2016. myPresto/omegagene: a GPU-accelerated molecular dynamics simulator tailored for enhanced conformational sampling methods with a non-Ewald electrostatic scheme. Biophysics and Physicobiology, $13,209-216$.

Lee, C., Cho, A. Y., Yoon, W., Yun, H., Kang, J and Lee, J. 2019. Co-crystal formation via resorcinol-urea interactions: Naringenin and carbamazepine. Crystal growth \& design, 19(7), 3807-3814.

Liu, X., Zhang, B., Jin, Z., Yang, H and Rao, Z. 2020. The crystal structure of 2019-NCoV main protease in complex with an inhibitor N3. RCSB Protein Data Bank.

Lopes, B., da Costa, M., Ribeiro, A., da Silva, T., Lima, C., Caruso, I and Desideri, A. 2020. Quercetin pentaacetate inhibits in vitro human respiratory syncytial virus adhesion. Virus Research, 276, 197805.

Mishra, S., Pandey, A and Manvati, S. 2020. Coumarin: An emerging antiviral agent. Heliyon, 6(1), e03217. 
Mohammad, F., Mohsin, A., Zaw, A., Tanveer, A., Waseem, A. 2020. Identification of Dietary Molecules as Therapeutic Agents to Combat COVID-19 Using Molecular Docking Studies, PREPRINT (Version 1) available at Research Square.

Narayanaswamy, R and Esa, N. 2018. Phytic acid (myo-inositol hexaphosphate)-a promising pharmaceutical agent: a review. Asian Journal of Pharmaceutical and Clinical Research, 11(11), 42-46.

Nile, S., Kim, D., Nile, A., Park, G., Gansukh, E and Kai, G. 2020. Probing the effect of quercetin 3-glucoside from Dianthus superbus L against influenza virus infection-In vitro and in silico biochemical and toxicological screening. Food and Chemical Toxicology, 135, 110985.

Patra, J., Das, G., Bose, S., Banerjee, S., Vishnuprasad, C., del Pilar Rodriguez, M and Shin, H. 2020. Star anise (Illicium verum):: Chemical compounds, antiviral properties, and clinical relevance. Phytotherapy Research. 1-20.

Plowe, C. 2005. Antimalarial drug resistance in Africa: strategies for monitoring and deterrence. In Malaria: drugs, disease and post-genomic biology (pp. 5579). Springer, Berlin, Heidelberg.

Reena Roy, D., Kandagalla, S and Krishnappa, M. 2020. Exploring the ethnomycological potential of Lentinus squarrosulus Mont. through GC-MS and chemoinformatics tools. Mycology, 11(1), 78.

Savarino, A., Lucia, M., Giordano, F and Cauda, R. 2006. Risks and benefits of chloroquine use in anticancer strategies. The Lancet. Oncology, 7(10), 792-793.

Sharma, A.D.; Kaur, I. 2020. Eucalyptol (1,8 cineole) from Eucalyptus Essential Oil a Potential Inhibitor of COVID 19 Corona Virus Infection by Molecular Docking Studies. Preprints 2020030455.

Shayeganmehr, A., Vasfi Marandi, M., Karimi, V., Barin, A and Ghalyanchilangeroudi, A. 2018. Zataria multiflora essential oil reduces replication rate of avian influenza virus (H9N2 subtype) in challenged broiler chicks. British Poultry Science, 59(4), 389-395.

Silva, A., Bento, C., Pena, A., Figueiredo, L., Prudêncio, C., Aguiar, L and Gomes, P. 2020. Cinnamic acid conjugates in the rescuing and repurposing of classical antimalarial drugs. Molecules, 25(1), 66.

Sotelo, J., Briceno, E and López-González, M. 2006. Adding chloroquine to conventional treatment for glioblastoma multiforme: a randomized, double-blind, placebo-controlled trial. Annals of Internal Medicine, 144(5), 337-343.

Sun, Y., Yang, A. W and Lenon, G. 2020. Phytochemistry, ethnopharmacology, pharmacokinetics and toxicology of Cnidium monnieri (L.) Cusson. International Journal of Molecular Sciences, 21(3), 1006.

Sun, N.; Wong, W.; Guo, J. 2020. Prediction of Potential 3CLpro-Targeting Anti-SARS-CoV-2 Compounds from Chinese Medicine. Preprints, 2020030247.

Tang, K., Zhang, X and Guo, Y. 2020. Identification of the dietary supplement capsaicin as an inhibitor of Lassa virus entry. Acta Pharmaceutica Sinica B.

Thomsen, R and Christensen, M. 2006. MolDock: a new technique for high-accuracy molecular docking. Journal of Medicinal Chemistry, 49(11), 3315-3321.

Uhlemann, A and Krishna, S. 2005. Antimalarial multi-drug resistance in Asia: mechanisms and assessment. In malaria: drugs, disease and post-genomic Biology (pp. 39-53). Springer, Berlin, Heidelberg.

Qamar, M., Alqahtani, S., Alamri, M and Chen, L. 2020. Structural basis of SARS-CoV-2 3CLpro and anti-COVID-19 drug discovery from medicinal plants. preprints.org.

Wang, J. 2020. Fast Identification of possible drug treatment of coronavirus disease-19 (COVID-19) through computational drug repurposing study. chemrxiv.org.

Wang, M., Cao, R., Zhang, L., Yang, X., Liu, J., Xu, M and Xiao, G. 2020. Remdesivir and chloroquine effectively inhibit the recently emerged novel coronavirus (2019-nCoV) in vitro. Cell Research, 30(3), 269-271.

Wang, B., Guo, H., Ling, L., Ji, J., Niu, J and Gu, Y. 2020. The Chronic Adverse Effect of Chloroquine on Kidney in Rats through an Autophagy Dependent and Independent Pathways. Nephron, 144(1), 53-64.

Wei, H., Wang, J., Cheng, C., Jin, L and Peng, J. 2020. Application of plant essential oils in pig diets. In feed additives (pp. 227-237). Academic press.

Xie, Y., Chen, Y., Guo, Y., Huang, Y and Zhu, B. 2020. Allicin and glycyrrhizic acid display antiviral activity against latent and lytic Kaposi's sarcoma-associated herpesvirus. Infectious Microbes \& Diseases.

Xu, X., Chen, P., Wang, J., Feng, J., Zhou, H., Li, X and Hao, P. 2020. Evolution of the novel coronavirus from the ongoing Wuhan outbreak and modeling of its spike protein for risk of human transmission. Science China Life Sciences, 63(3), 457-460.

\section{Figures}


A



Capsaicin $+3 \mathrm{CL}$-protease
B

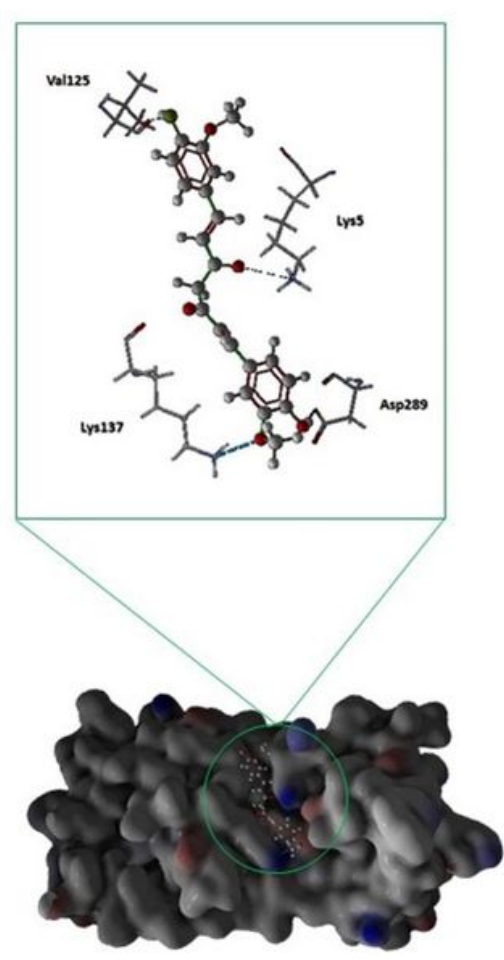

Curcumin + 3CL-protease

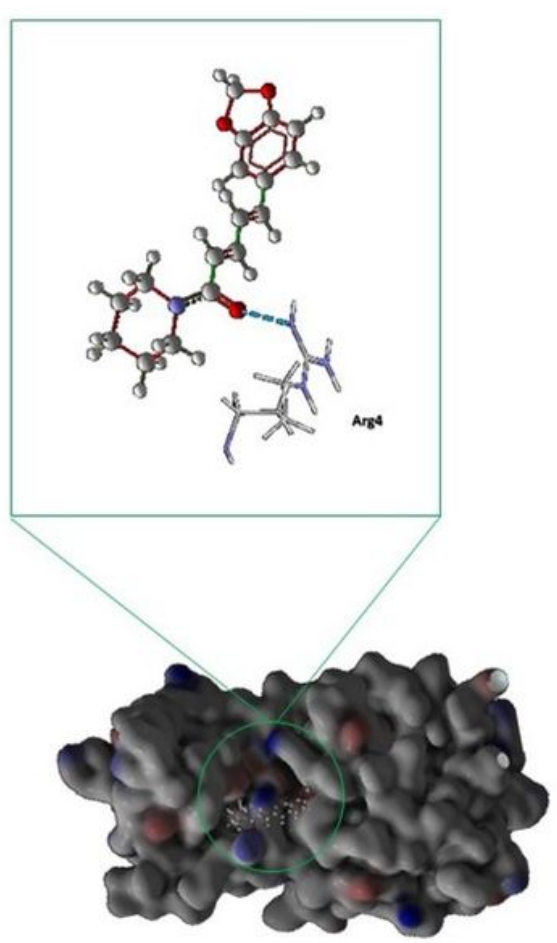

Piperin +3 CL-protease

Figure 1

Visualization of the result of 3CL protease-phytochemical docking. The results of the docking of the three phytochemical compounds with better interaction energies than the antimalarial drugs considered in this study are shown. A) capsaicin, the interaction by hydrogen bridge with Asp 153 and by steric interaction with Lys 102 is shown; B) curcumin, the interaction by hydrogen bridge with Val 125, Asp 289 and Lys 137 and by steric interaction with Lys 5 is shown; C) piperin, the interaction by hydrogen bridge and by steric interaction only with Arg4 is shown.

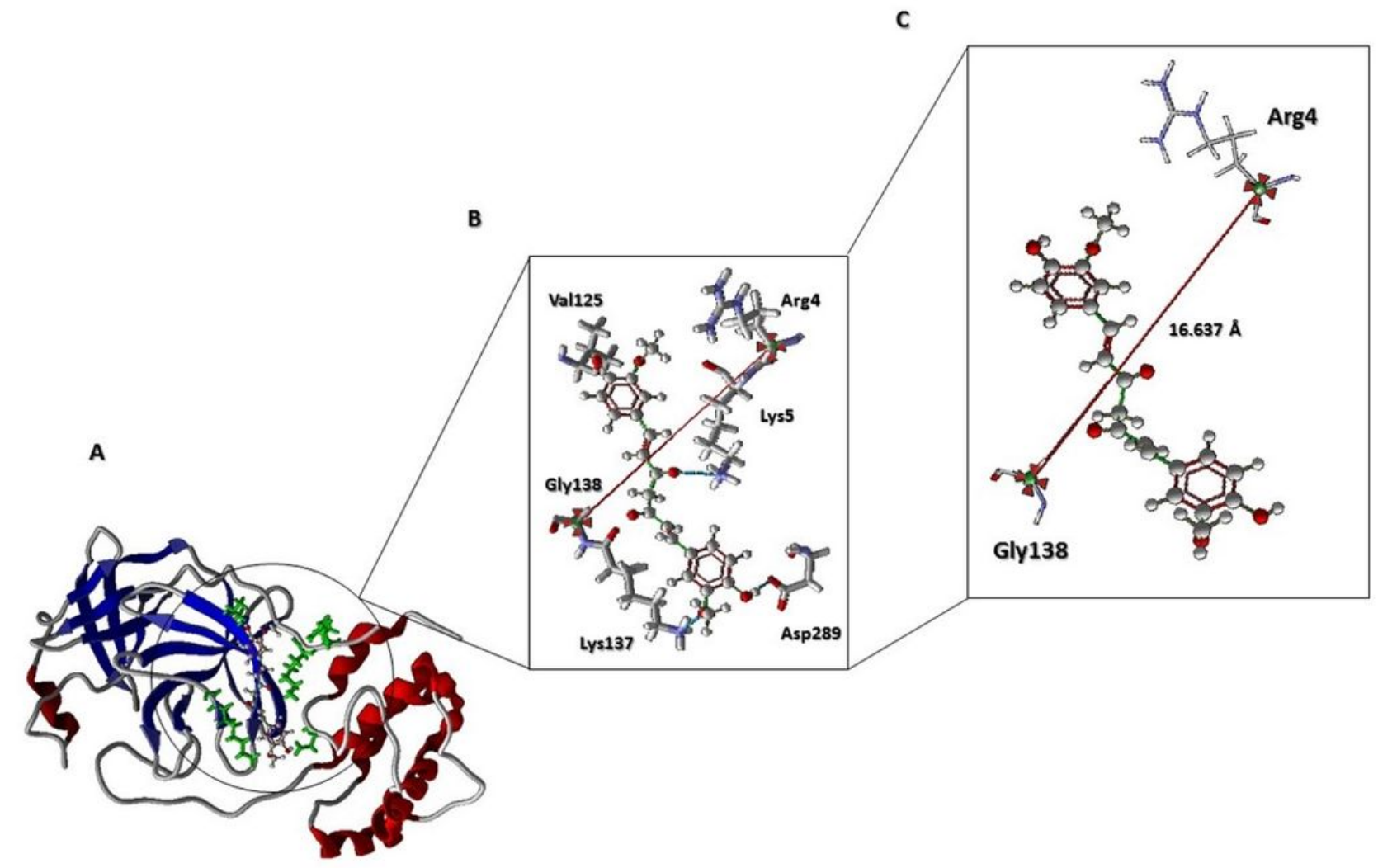


Figure 2

The location and distance between residues Arg 4 and Gly 138 are shown. They are approximately $16 \AA$ apart from each other and at approximately $2 \AA$ distance from almost all the ligands (except capsaicin, which presents a thermodynamically more favorable coupling in a cavity that bets for the one planned for the rest of the ligands). These residues were arbitrarily chosen to measure the conformational perturbation of the protease in the presence of each ligand.

A) the figure shows the $3 \mathrm{CL}$ protease-curcumin docking as reference, $\mathrm{B}$ ) as well as all the residues close to the ligand and C) the distance between the residues chosen for the MD analyzes.

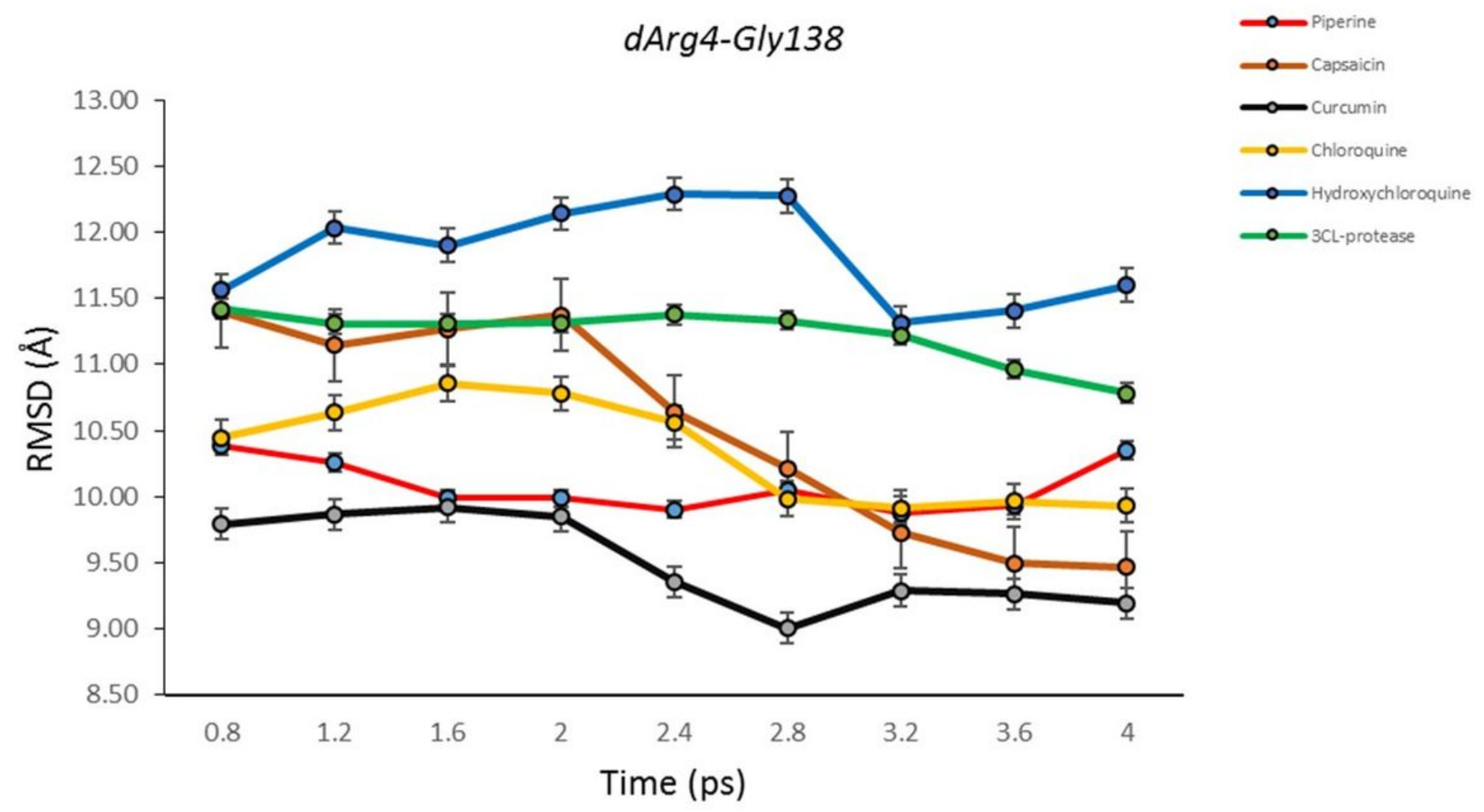

Figure 3

Conformational fluctuation and partial folding/unfolding of $3 \mathrm{CL}$-protease in the presence of compounds and as a function of time. Using as a reference the distances between the residues of Arg4 and Gly138.

\section{Supplementary Files}

This is a list of supplementary files associated with this preprint. Click to download.

- InformationSupplementaryTheoreticalMolecularDockingStudyoftheStructuralDisruptionoftheViral3CLProteaseofCOVID19InducedbyBindingofCapsaicinPi 